\title{
BMJ Open Referrals for uncomplicated lower back pain: a cluster parallel randomised trial of patient-centred communication to improve the management of acute back pain in primary care. A study protocol
}

To cite: Schedlbauer A, Burggraf L, Hueber S, et al. Referrals for uncomplicated lower back pain: a cluster parallel randomised trial of patient-centred communication to improve the management of acute back pain in primary care. A study protocol. BMJ Open 2019;9:e027718. doi:10.1136/ bmjopen-2018-027718

- Prepublication history for this paper is available online. To view these files, please visit the journal online (http://dx.doi. org/10.1136/bmjopen-2018027718).

Received 09 January 2019 Revised 14 August 2019 Accepted 02 September 2019

\section{Check for updates}

C) Author(s) (or their employer(s)) 2019. Re-use permitted under CC BY-NC. No commercial re-use. See rights and permissions. Published by BMJ.

${ }^{1}$ Insitute of General Practice, Friedrich-Alexander-Universität Erlangen Nürnberg (FAU), Universitätsstraße 29, Erlangen, Germany

${ }^{2}$ Institute of Clinical Psychology, Friedrich-Alexander-Universität Erlangen Nürnberg (FAU), Nägelsbachstrasse 25a, Erlangen, Germany

Correspondence to Dr Angela Schedlbauer; angela.schedlbauer@ukerlangen.de

\section{ABSTRACT}

Introduction Low back pain (LBP) is one of the most frequent encounters in General Practice. Investigation and referral remain common despite the self-limiting character of episodes that are not largely attributable to specific underlying injuries. Identifying patients' ideas, concerns and expectations (ICE) is a well-established element within consultation skills training and has been shown to improve prescribing. It can be a powerful communication tool setting the base for transferring and adjusting adequate clinical information. This study aims to evaluate whether ICE can decrease unnecessary medicine in the management of acute LBP in primary care.

Methods and analysis Research question: Does ICE training intervention have an effect on doctors' referrals of patients suffering from acute LBP? Population: Recruitment to this parallel cluster randomised trial will take place among general practitioners belonging to four independent practice networks in Northern Bavaria/Germany. Intervention: At baseline, 24 out of 48 doctors will be randomly assigned to take part in a 1-day training session covering theoretical background and clinical implementation of patientcentred communication by stimulating ICE. They will also be given access to a web-based supporting tool for reflective practice on their communication skills. Comparison: GPs in the control group will continue consultations as usual. Outcome: Outcome measures are referrals to diagnostic imaging, physiotherapy and specialists obtained from routine practice data, compared between intervention and control group. Time: Referrals of patients consulting their doctors for documented LBP will be monitored up to 3 months after the ICE training intervention.

Ethics and dissemination Ethical approval for the study was obtained by the Ethics Committee of the University Erlangen-Nuremberg (296_17B). Results will be disseminated by conference presentations and journal publications.

Trial registration number The trial is registered in clinicaltrials.gov (NCT03711071).

\section{Strengths and limitations of this study}

- A thorough randomised parallel design was chosen to compare the effect of patient-centred communication training on doctors' clinical behaviour in the management of acute low back pain.

- The practice networks included in the trial involve a broad spectrum of urban and rural practices being representative for German primary care.

- Blinding is more difficult to achieve in non-pharmacological trials and represents a clear limitation of this study. Masking participating doctors towards treatment allocation is attempted by allocating the behavioural intervention at two stages: the intervention group will undergo the ideas, concerns and expectations (ICE) training session at baseline and the control group will be offered training once data collection is accomplished. An effort will be made to hide outcome measures from all participants.

- The difficulty of monitoring ICE communication technique during consultations without introducing a considerable threat of bias (performance and reporting bias) is a clear limitation of this study. The effect of one ICE training session on doctors' behaviour focused on in this study can only act as a proxy for using more ICE when communicating with patients.

- The innovative character of this research project bears its risks. In Bavaria/South Germany, this is the first project establishing a practice network for research purposes in primary care and the feasibility of a randomised controlled trial in this setting will need to be tested.

\section{INTRODUCTION}

Back pain is one of the most frequent reasons for encounter in General Practice. ${ }^{12}$ It affects all ages with a peak in prevalence in the fifth decade and a decrease in the sixth and seventh decades of life. In a German Health Survey, as many as $39 \%$ of female and $31 \%$ of male participants aged 18-80 reported episodes of 
back pain within the last 7 days. ${ }^{3} \mathrm{~A}$ multiregional study conducted in Germany confirmed a social and educational impact revealing higher prevalence in patients with poor education ( $47 \%$ in patients with 9 years of education or less compared with $27 \%$ with more than 12 years of education). ${ }^{4}$ Patients suffering from back pain were also shown to have increased levels of comorbidity. ${ }^{5-12}$

Reasons for low back pain (LBP) often remain unspecific with $80 \%-90 \%$ of cases not being attributable to a specific injury or lesion. ${ }^{13}$ The majority of episodes are self-limiting, of which $90 \%$ show spontaneous remissions within 6 weeks. Only less than $7 \%$ of episodes remain chronic. ${ }^{1}$ Nevertheless, investigation and referral remain common and back pain clearly represents a condition contributing to unnecessary healthcare and costs. These are responsible for expenditures in the dimension of $€ 8$.4 billion per year in Germany, of which $15 \%$ are contributable to expenses for medical interventions and $85 \%$ to periods of unemployment and resulting loss of productivity. ${ }^{3}$

Insufficient information regarding diagnostic and therapeutic procedures can be the cause of false patient expectations. Communication is a simple but powerful tool with a great potential of transferring relevant and adequate information, and patient-centred communication has been shown to influence patient contentedness and adherence to medical treatment. ${ }^{14-16}$ However, patients' frequent complaint of poor communication and inadequate treatment is an indication for a clear mismatch between patients' and physicians' concerns. ${ }^{17-23}$ Patient-centred communication can be promoted by ICE, an easily applicable and internationally approved communication technique that encourages patients to disclose their ideas, concerns and expectations (ICE) within a consultation. ${ }^{24}$ Despite increasing evidence of a positive influence on health-related outcomes such as improved communication skills and medication prescribing, ${ }^{17}$ the implementation of ICE in clinical routine is still lacking; this may be because physicians view patient-centred communication as being time-intensive and cost-intensive. ${ }^{25}$

Unnecessary medicine often emerges from discrepancy between clinical facts and patients' expectations, resulting in irrelevant healthcare with a high amount of direct and indirect economic losses. A recent cluster randomised trial confirmed clinical benefit of a cognitive education programme for patients with $\mathrm{LBP}^{26}$ In this research project, we will evaluate whether the implementation of the ICE technique can contribute to more sensible resource allocation and less unnecessary medicine in the management of acute LBP in primary care.

\section{METHODS AND ANALYSIS \\ Aims}

The overall purpose of the proposed study is to carry out a patient-centred communication training based on ICE technique and to evaluate its ability of preventing unnecessary medicine. We would like to know whether ICE is applicable in daily routine, enhances patient satisfaction during consultations and results in a reduction of diagnostic imaging and specialist referring with a positive impact on financial resources by reducing direct and indirect healthcare costs.

The primary aim to be achieved is to examine the effect of doctors' ICE training on patient referrals for acute LBP. ICE communication training will be developed and administered within the network of research practices. Patient attitudes regarding their physical indisposition and possible diagnostic and treatment options will be addressed by exploring ICE. Subsequent consultations of patients presenting with uncomplicated LBP will be monitored in terms of referrals to further imaging, physiotherapy or specialist treatment.

The secondary aim of this project is to assess how ICE communication can influence consultation quality based on doctors' and patient views, and will be reported elsewhere.

\section{Trial design and setting}

The design of a randomised trial of non-pharmacological treatment applying parallel cluster randomisation has been chosen to overcome the difficulty of allocating doctors working in the same practice to different interventions without running the risk of contamination. ${ }^{27}$ Trials of nonpharmacological treatment test complex behavioural interventions involving several components that are difficult to describe, standardise and administer consistently to all patients. To overcome these difficulties, the rules of the Consolidated Standards of Reporting Trials (CONSORT) Statement of nonpharmacological treatments were applied in the design of the proposed study. ${ }^{28}$ The observations of patients treated for acute LBP by the same doctor may be correlated or clustered. Each doctor taking part in this trial forms a cluster of clinical treatment decisions being similar and not independent from one another.

\section{Participant recruitment}

The clusters will involve two levels: doctors recruited to receive training in patient-centred ICE communication (intervention group) or no training (control group), and patients being treated by these doctors for acute LBP.

General practitioners (GPs) will be randomised to receive the intervention or not. They will be recruited from four independent practice networks forming 'Forschungspraxen Franken', a newly setup research network located in rural and urban areas of Franconia/ Northern Bavaria comprising 119 GPs from 77 practices, of which 30 are single-handed and 47 are group practitioners.

Patients aged 18 and above with a new episode of unspecific LBP, defined as no prior visit for LBP within the previous 6 weeks, will be included. Patients consulting their doctors for LBP will be identified via the practice electronic health records. Inclusion criteria are consultations involving patients over 18 years of age consulting their doctor for uncomplicated acute LBP based on the 
following ICD codes: M43.19, M54.05, M54.06, M54.07, M54.08, M54.15, M54.16, M54.17, M54.18, M54.3, M54.4, M54.5, M54.85, M54.86, M54.87, M54.88, M54.89, M54.95, M54.96, M54.97, M54.98, M54.99. According to the definitions of acute LBP in the national German guidelines, ${ }^{29}$ inclusion will be restricted to patients not having consulted their GPs for back problems within the last 6 weeks. Patients with diagnoses of specific back pain such as disc prolapse, vertebral body fracture or malignant disease, either already known or added during the observation period, will be excluded. Please see a more detailed description under data collection and management.

\section{Allocation sequence generation and randomisation}

At baseline, 48 GPs will be randomised to receive the intervention or not, with participating doctors acting as units of randomisation and analysis. To minimise contamination in the control group, randomisation to ICE training intervention will take place at the practice level with units of randomisations being single-handed or group practices belonging to the research network 'Forschungspraxen Franken'. This ensures that doctors being allocated to the control group will not be surrounded by colleagues having received the ICE training intervention.

Stratification will be imposed on the randomisation process to minimise numeral imbalance over treatment groups during the course of randomisation. As all practices will be available at the time of generating the sequence, the random allocation rule can be applied. Retrospectively, the possible occurrence of selection bias will be assessed by identifying the number of participants initially recruited as well as those actually included, and by comparing characteristics of individuals between intervention and control group at baseline.

To ensure numeral balance of the number of individuals randomised to each group, practices will be stratified by numbers of GPs per practice. The sequence will be generated independently within each stratum. For example, in a stratum with a sample size of six practices comprising four GP partners each, three practices will be allocated to the control group and three will be allocated to the intervention group by drawing six concealed envelopes containing three group A and three group B allocations without replacing them, thus allocating three of the six practices to the control group and three to the intervention group. ${ }^{30}$

\section{Blinding}

The fact that blinding of participating doctors towards the intervention will not be possible represents a considerable threat of bias. However, the following attempts will be undertaken to hide from participating doctors who received true treatment allocation: First, all participants will be blinded towards the explicit purpose and design of the study. Second, ICE communication training will be offered to all participants: to GPs in the intervention group as a true intervention at the beginning of the RCT and to control GPs as a pretend intervention at the end of the trial. The treatment allocation for each site and each doctor will be kept at the Insititute of General Practice in Erlangen. The invitation for the communication training will be sent to both intervention and control practices, but control practices will have their training at a later point in time. Neither practices and GPs forming the clusters, nor healthcare assistants involved with data collection or patients seeing their GPs for backache will beaware of the intentional delay. Even though it will not be possible to blind doctors towards having received ICE communication training, this approach attempts to blind those supposed to administer the ICE communication (the doctors) and those responsible for data entry and processing (the receptionists) towards the true treatment intentions and study outcomes. Due to the anonymised nature of patient data collected for referral outcomes, patients will not need to be made aware of the research project.

\section{Interventions}

The ICE training intervention consists of a 1-day educational session for doctors encouraging reflection on actual doctor-patient communication and introducing the theoretical background of ICE. In close association with recommendations of the national guidelines for acute $\mathrm{LBP}^{29}$ the training provides clues on how to encourage patients to report their ICE and offers communication skills training through standardised patient scenarios.

Furthermore, a web-based supporting tool tailored towards participating doctors' needs will be implemented. Doctors will be given access to a password-protected online platform that holds a summary of the training session content for personal reference, as well as an interactive practice game to help doctors reflect on their individual communication style. The platform will also feature a questionnaire about attitudes towards the ICE concept that doctors can fill online for self-reflection.

\section{Outcomes}

The primary outcome measure consists in GP referrals in the weeks and months following the ICE training intervention, involving referrals to diagnostic imaging, physiotherapy, specialist neurologists, orthopaedic surgeons and hospital admissions. They will pertain both to the cluster level (doctors' decisions) and the individual level (patient referrals). Sickness absence from work will be the secondary outcome measure.

Associating factors for referrals will be considered including patient age, gender and co-morbidities (depression, chronic back pain), as well as doctors' age, gender and practice characteristics (rural, urban).

The patient and doctor perspective will be evaluated via questionnaires and interviews based on a qualitative study design which is not part of this study protocol, but which will be reported separately. 


\section{Sample size}

Sample size calculations endorse the necessary inflation of the sample size due to the cluster design, allowing for each doctor forming a cluster of clinical decisions that contain similar treatment decisions not independent from one another. Sample sizes also take into consideration the intracluster correlation coefficient, the number of events, the expected effect and the power of the study. Assuming a referral rate of $30 \%$ for acute LBP, as reported in German routine data, ${ }^{31}$ an absolute alteration in referring patients in the magnitude of $10 \%$ was considered as clinically relevant. Presuming an intracluster correlation coefficient (ICC) of 0.05 , a significance level of 0.05 and a power of $0.8,24$ GPs seeing 40 patients each will be needed in each study group to detect a decrease in referrals from $30 \%$ in the control group to $20 \%$ or less in the intervention group. Alternative sample size requirements will be considered based on actual referral rates.

\section{Data analysis}

Data analysis will compare referral in the intervention and treatment group in terms of the proportion of patients referred for further diagnostics or treatment out of all patients consulting for LBP. The effect of ICE training will be estimated through logistic regression, examining the association between the ICE training intervention (the main explanatory variable) and further referrals (main outcome variable). Random effects logistic regression will be applied to evaluate the influence of other factors such as patient, doctor and practice characteristics.

A per-protocol analysis will be applied, including only participants attending the ICE training intervention in order to focus on the effect of the ICE training. This approach is justified by intervention assignment taking place in a blinded manner prior to the analysis.

\section{Data collection and management}

Data collection will focus on consultations for unspecific acute LBP and starts once doctors from the intervention group have completed the ICE training session. Patients consulting the participating doctors for acute LBP will be identified via the practice electronic health records and routinely generated clinical data will be collected retrospectively. A trained practice healthcare assistant will extract the predefined outcome and exposure variables described above in a standardised data collection sheet. According to sample size requirements, data collection will involve 40 consecutive patients fulfilling the inclusion criteria. Anticipating about four consultations for uncomplicated LBP per GP per week, data collection will continue up to 12 weeks post intervention until data of 40 consultations will have been collected for each of the 48 GPs, resulting in a final number of 1920 of consultations.

A modified CONSORT flow diagram will be provided to describe and specify number of practices, doctors and patients throughout the different stages of the trial.

\section{Patient and public involvement}

Patients were not involved in the development of the research question nor in the design of the study. The intervention was considered to involve no burden to patients. However, patients' involvement and their perspectives will play an important role in interview and questionnaire-based surveys that will be described elsewhere. The outcomes of this research project will be disseminated to participating practices and their patients.

\section{Trial monitoring}

Quality assurance is attempted through methodological rigour, keeping all possible biases to a minimum. The centre for clinical studies monitoring the study's progress regularly will have to ensure that the methodology is applied adequately.

\section{Ethics and dissemination}

Patient data collection will take place by a dedicated member of staff within a health centre assuring patient anonymity of the collected data. Informed Consent will therefore not be required. The data sheets being transferred to the Institute of General Practice at the University of Erlangen-Nürnberg will not allow detecting patient identity.

Results from this study will be published in peer-reviewed scientific journals according to reporting guidelines and presented at conferences.

Contributors AS: Study design, methods, writing original draft. LB: Project management, theoretical development, methodological adjustment. SH: Study design, methods, review and editing. I-AT-S: Project partner, concept and development of interactive online tool for reflective practice. MR: Study design, methods, supervision, review and editing. TK: Study design, supervision, review and editing.

Funding This study was funded by the German Federal Ministry of Education and Research (Bundesministerium für Bildung und Forschung, BMBF), grant number $01 \mathrm{GY} 1605$.

\section{Competing interests None declared.}

Patient consent for publication Not required.

Ethics approval Ethic approval was received by the institutional review board of Erlangen University ethic commission (Ethikkommission der Friedrich-AlexanderUniversität Erlangen-Nürnberg, Erlangen, Germany; Ethics Committee of the University Erlangen-Nuremberg, Erlangen/Germany: Number 296_17 B).

Provenance and peer review Not commissioned; externally peer reviewed.

Open access This is an open access article distributed in accordance with the Creative Commons Attribution Non Commercial (CC BY-NC 4.0) license, which permits others to distribute, remix, adapt, build upon this work non-commercially, and license their derivative works on different terms, provided the original work is properly cited, appropriate credit is given, any changes made indicated, and the use is non-commercial. See: http://creativecommons.org/licenses/by-nc/4.0/.

\section{REFERENCES}

1 Andersson G. The epidemiology of spinal disorders. The adult spine: Principles and practice, 1997: 93-141.

2 Deyo RA, Mirza SK, Martin BI. Back pain prevalence and visit rates: estimates from US national surveys, 2002. Spine 2006;31:2724-7.

3 Gesundheit in Deutschland. Berlin: Robert Koch-Institut 2006:117-8.

4 Schmidt CO, Raspe H, Pfingsten M, et al. Back pain in the German adult population: prevalence, severity, and sociodemographic correlates in a multiregional survey. Spine 2007;32:2005-11. 
5 Schneider S, Mohnen SM, Schiltenwolf M, et al. Comorbidity of low back pain: representative outcomes of a national health study in the federal Republic of Germany. Eur J Pain 2007;11:387-97.

6 Hagen EM, Svensen E, Eriksen HR, et al. Comorbid subjective health complaints in low back pain. Spine 2006;31:1491-5.

7 Hestbaek L, Leboeuf-Yde C, Kyvik KO, et al. Comorbidity with low back pain: a cross-sectional population-based survey of 12- to 22-year-olds. Spine 2004;29:1483-91.

8 Hestbaek L, Leboeuf-Yde C, Manniche C. Is low back pain part of a general health pattern or is it a separate and distinctive entity? A critical literature review of comorbidity with low back pain. $J$ Manipulative Physiol Ther 2003;26:243-52.

9 Currie SR, Wang J. Chronic back pain and major depression in the general Canadian population. Pain 2004;107:54-60

10 Polatin PB, Kinney RK, Gatchel RJ, et al. Psychiatric illness and chronic low-back pain: the mind and the spine-which goes first? Spine 1993;18:66-71.

11 Ritzwoller DP, Crounse L, Shetterly S, et al. The association of comorbidities, utilization and costs for patients identified with low back pain. BMC Musculoskelet Disord 2006;7:72.

12 Schur EA, Afari N, Furberg $\mathrm{H}$, et al. Feeling bad in more ways than one: comorbidity patterns of medically unexplained and psychiatric conditions. J Gen Intern Med 2007;22:818-21.

13 Chou R, Fu R, Carrino JA, et al. Imaging strategies for low-back pain systematic review and meta-analysis. The Lancet 2009;373:463-72.

14 Mead N, Bower P, Hann M. The impact of general practitioners' patient-centredness on patients' post-consultation satisfaction and enablement. Soc Sci Med 2002;55:283-99.

15 Dwamena F, Holmes-Rovner M, Gaulden CM, et al. Interventions for providers to promote a patient-centred approach in clinical consultations. Cochrane Database Syst Rev 2012;48.

16 Epstein RM, Franks P, Shields CG, et al. Patient-centered communication and diagnostic testing. Ann Fam Med 2005:3:415-21.

17 Matthys J, Elwyn G, Van Nuland M, et al. Patients' ideas, concerns, and expectations (ICE) in general practice: impact on prescribing. $\mathrm{Br}$ $J$ Gen Pract 2009;59:29-36.

18 Böcken J, Braun B. Reipschläger U. Gesundheitsmonitor 2011: Bürgerorientierung Im Gesundheitswesen-Kooperationsprojekt Der Bertelsmann Stiftung und Der BARMER GEK: Verlag Bertelsmann Stiftung 2012.
19 Keitz SA, Stechuchak KM, Grambow SC, et al. Behind closed doors: management of patient expectations in primary care practices. Arch Intern Med 2007;167:445-52.

20 Lado E, Vacariza M, Fernández-González C, et al. Influence exerted on drug prescribing by patients' attitudes and expectations and by doctors' perception of such expectations: a cohort and nested casecontrol study. J Eval Clin Pract 2008;14:453-9.

21 Little P, Dorward M, Warner G, et al. Importance of patient pressure and perceived pressure and perceived medical need for investigations, referral, and prescribing in primary care: nested observational study. BMJ 2004;328.

22 van Driel ML, De Sutter A, Deveugele M, et al. Are sore throat patients who hope for antibiotics actually asking for pain relief? The Annals of Family Medicine 2006;4:494-9.

23 Cartwright A. General practice revisited. Psychological Medicine 1981;11:870.

24 Pendleton D, Schofield T, Tate P, et al. The new consultation: developing doctor-patient communication. Oxford University Press, 2003.

25 van den Eertwegh V, van Dulmen S, van Dalen J, et al. Learning in context: identifying gaps in research on the transfer of medical communication skills to the clinical workplace. Patient Educ Couns 2013;90:184-92.

26 Werner EL, Storheim K, Løchting I, et al. Cognitive patient education for low back pain in primary care: a cluster randomized controlled trial and cost-effectiveness analysis. Spine 2016;41:455-62.

27 Kirkwood B, Sterne J. Essential medical statistics (essentials. 2nd edn. Wiley-Blackwell, 2003.

28 Boutron I, Altman DG, Moher D, et al. Consort statement for randomized trials of nonpharmacologic treatments: a 2017 update and a consort extension for nonpharmacologic trial Abstracts. Ann Intern Med 2017;167:40-7.

29 Chenot J-F, Greitemann B, Kladny B, et al. Non-Specific low back pain. Dtsch Arztebl Int 2017;114:883-90.

30 Schulz KF, Grimes DA. Generation of allocation sequences in randomised trials: chance, not choice. The Lancet 2002;359:515-9.

31 Horenkamp-Sonntag D, Linder R, Engel S. Nutzung von AU-Daten Zur tagesgenauen Bestimmung von ICD-Diagnosen Im ambulanten Bereich am Beispiel Rückenschmerz. 7. Freiburg: AGENSMethodenworkshop, 2015. 